\title{
OPTIMAL CONTROL OF THE HYDRAULIC ACTUATED BOOM SYSTEM BASED ON PORT-HAMILTONIAN FORMULATION
}

\author{
Lingchong $\mathrm{Gao}^{1 *}$, Boyang Shi ${ }^{1,2}$, Micheal Kleeberger ${ }^{1}$, Johannes Fottner ${ }^{1}$ \\ ${ }^{I}$ Chair of Materials Handling, Material Flow, Logistics, Technical University of Munich, Boltzmannstr. 15, 85748, \\ Garching, Germany \\ ${ }^{2}$ Department of Engineering Mechanics, Dalian University of Technology, Linggong Road No.2, 116085, Dalian \\ V.R China \\ * Corresponding author: Tel.: +49(89)351-15926; E-mail address: lingchong.gao@tum.de
}

\begin{abstract}
The boom systems of mobile cranes and aerial platform vehicles are driven by hydraulic systems, to be specified, valve-controlled hydraulic cylinders. This hydraulic actuated boom system can accomplish the tasks such as lifting heavy loads or carrying personal to high position, by the design of a long boom structure. In practice, the boom structure is designed as light and slender as possible to control the structure self-weight. However, such structure is quite flexible and can be easily stimulated by the loads, including the driving force or torque from the hydraulic system. Our research focuses on trajectory planning for hydraulic actuated boom where both hydraulic driven system and boom structure deformation are considered. In this paper, the hydraulic actuated boom system is formulated as a port-Hamiltonian system which is a proper modelling method for multi-domain system. The problems of trajectory optimization and vibration control are formulated as optimal control problem based on port-Hamiltonian model and this procedure is tested on a model of hydraulic cylinder. A reasonable result is solved with the selected cost function and inputs.
\end{abstract}

Keywords: Hydraulic Actuated Boom, Optimal Contorl, Port-Hamiltonian

\section{INTRODUCTION}

The hydraulic actuated boom system is usually equipped by hydraulic mobile cranes and aerial platform vehicles to enable the ability of changing the configuration between operation status and mobile or transportation status. Besides, mounted with a complete hydraulic system which provides a stable and powerful energy source, the boom systems can handle heavy tasks such as lift and transport heavy payloads or personals to specified location or height.

The analysis of hydraulic actuated boom system including the elasticity of both system, hydraulic and boom structure, has drawn many attentions in the resent years. The researchers are interested to investigate the method to control the vibration respond of the hydraulic actuated boom system. Someone focuses on the active damper for the structure, some others pay attention on the fluctuation reduction of the hydraulic system. Sun combined mathematical formulations of hydraulic drive system with the finite element model of the boom structure, and formulated a complete model to describe the dynamic interaction between the boom structure and the drive system of mobile crane[1]. This method has been applied solve the dynamic calculation of slewing, lifting and luffing operations of lattice boom cranes[2][3]. Besides the mobile cranes and the aerial platform vehicles, fire-rescue turntable ladders also use such similar hydraulic actuated boom system. Prof. Sawodny and his team from Uni. Stuttgart has studied the active vibration control problem of this ladder system for more than ten years. They started with a discretion of the long fire-rescue turntable ladder as a flexible multi-body system[4] and the hydraulic drive system was included in the mathematical model as set of equations [5]. In the work of [6], the fire-rescue turntable ladder was built as a distributed- parameter model of EulerBernoulli beam and transferred into a low dimensional model space. In their recent work[7], a 3-dimensional model of ladder was derived to describe the coupled bending-torsional vibration 
associated with the slewing operation and an active vibration damping control was developed with validation in real operation.

\section{METHODOLOGY}

\subsection{Port-Hamiltonian system}

The definition of port-Hamiltonian system is related with a space of power variables that are strictly connected with the geometry structure of the system. A series of geometric structures defined on this space are necessary to describe the different parts of the system in different domains separately and the internal or external interconnection of the whole system. These geometric structures are defined as Dirac structure which is the key mathematical concept to connect multi-domain system in a unified description. According to the definition given by Duindam[8], we give a linear space $\mathcal{F}$ (space of flow or velocity) and its dual denotes as $\mathcal{E}=\mathcal{F}^{*}$ (space of effort or force). And a Dirac structure on $\mathcal{F}$ is a linear subspace $\mathcal{D} \in \mathcal{F} \times \mathcal{E}$ with the property $\mathcal{D}=\mathcal{D}^{\mathrm{T}}$.

The Hamiltonian function, as the total energy function of the system, is used to illustrate the relation between the pair of flow and effort variables. We define the time derive of the state variables as the general flow variables as $f=\dot{x}$, and define the co-energy variables as the general effort variables as $e=\partial H / \partial x$. Then the portHamiltonian $(\mathrm{PH})$ representation of state space model can be given in the $\mathrm{I} / \mathrm{O}$ form of

$\dot{\boldsymbol{x}}=(\boldsymbol{J}(x)-\boldsymbol{R}(x)) \frac{\partial H}{\partial x}+\boldsymbol{G}(x) \boldsymbol{u}$

$\boldsymbol{y}=\boldsymbol{G}(x)^{\mathrm{T}} \frac{\partial H}{\partial x}+\boldsymbol{D} \boldsymbol{u}$

with $\boldsymbol{J}(x)$, a skew-symmetic matrix, and the symmetric and positive semi-definite matrix $\boldsymbol{R}(x)$ which represents the passive part in the system.

For our tasks, at first, we have to consider the hydraulic actuated boom system separately, as boom part and hydraulic part. Many works have been accomplished related with the modelling and control of the flexible boom structure within the framework of port-Hamiltonian system. The pot-Hamiltonian model of Timoshenko beam was reformulated by Macchelli[9]. And the similar approach for multi-body system, including rigid body, flexible body and kinematic pairs, was also developed based on the idea of power conserving interconnection[10]. In order to transfer the model of distributed Timoshenko beam into a solvable discretized model, a discretization method which can preserve the geometric structure of the system is developed to preserve the property of (Stokes-) Dirac structure in the discretized finite-dimensional model[11][12]. Wang applied a geometric pseudo-spectral discretization to obtain the finite-dimensional Port-Hamiltonian framework of plana Timoshenko beam model, and solved the feedforward motion control problem based on this lumped model [13]. For the hydraulic systems, Kugi designed a nonlinear controller for a system of hydraulic cylinder based on the PortHamiltonian model [14]. Grabmair designed an energy-based nonlinear controller for the hydraulic actuated wrapper assembly by using the port-Hamiltonian formulation of hydraulic cylinder[15]. Sakai developed passivity based control for a hydraulic robot arm system based on the port-Hamiltonian model of the hydraulic cylinder[16][17]. He also applied Casimir function to improve computation efficiency[18], and developed a nondimensionalization method which can preserve the parametric structure within the framework of port-Hamiltonian system to reduce the size of parameter space[19]. There is also some research related with the combination of hydraulic system and flexible boom system. Stadlmayr presented a portHamiltonian representation of flexible manipulator consisting with a long boom with a mass at the tip and hydraulic cylinder. Combined with feed-forward and feedback control system, a MIMO-control was designed to accomplish path tracking and vibration suppression for the flexible manipulator[20].

\subsection{Problem formulation of optimal control}

In this paper, the optimal control problem for the hydraulic system can be described as the following general optimal control problem:

$$
\begin{gathered}
\min J=\int_{t_{0}}^{t_{\mathrm{f}}} f_{0}(\boldsymbol{x}(t), \boldsymbol{u}(t), t) \mathrm{d} t \\
\text { s.t. } \dot{\boldsymbol{x}}(t)=\boldsymbol{f}(\boldsymbol{x}(t), \boldsymbol{u}(t), t) \\
\boldsymbol{x}\left(t_{0}\right)=\boldsymbol{x}_{0}, \boldsymbol{x}\left(t_{\mathrm{f}}\right)=\boldsymbol{x}_{\mathrm{f}} \\
\boldsymbol{h}(\boldsymbol{x}) \leq \mathbf{0} \\
\boldsymbol{g}(\dot{\boldsymbol{x}}) \leq \mathbf{0}
\end{gathered}
$$

where $\boldsymbol{x}(t) \in \mathbb{R}^{n_{x}}$ are state variables, $\boldsymbol{u}(t) \in$ $\mathbb{R}^{n_{u}}$ are control inputs. $J$ is a Lagrangian-type 
cost functional where $f_{0} \in \mathbb{R}^{n_{x}} \times \mathbb{R}^{n_{u}} \times \mathbb{R}^{n_{u}} \rightarrow$ $\mathbb{R}$ is an integral term which is chosen to reflect the response of vibration or/and energy consumption. Differential equations $\dot{\boldsymbol{x}}(t)=$ $\boldsymbol{f}(\boldsymbol{x}(t), \boldsymbol{u}(t), t)$ denote dynamics of the system. Boundary conditions $\boldsymbol{x}\left(t_{0}\right)=x_{0}, \boldsymbol{x}\left(t_{\mathrm{f}}\right)=x_{\mathrm{f}}$ are initial state variables and terminal variables, respectively. $\boldsymbol{h}(\boldsymbol{x}) \leq \mathbf{0}$ denotes inequality constraints with respect to state variables $\boldsymbol{x}$. $\boldsymbol{g}(\dot{\boldsymbol{x}}) \leq \mathbf{0}$ denotes inequality constraints with respect to rates of state variables $\dot{\boldsymbol{x}}$. The aim is to find optimal control inputs $\boldsymbol{u}^{*}(t)$ and state variables $\boldsymbol{x}^{*}(t)$ which minimize the cost functional and fulfil the differential equations, boundary conditions, and all inequality constraints.

To solve the optimal control problem Eq.(2) numerically, the software package ICLOCS 2.5 [21][22] is applied in this paper. In the ICLOCS 2.5 , the direct collocation method is chosen to transform the optimal control problem (2). The main idea can be described as follows. First, the time domain $\left[t_{0}, t_{\mathrm{f}}\right]$ is divided into $N$ intervals with $\mathrm{N}+1$ time grids $\left\{t_{0}, t_{1}, \ldots, t_{1}, \ldots, t_{N}=t_{\mathrm{f}}\right\}$. The state variables and control inputs on grids $\left\{\boldsymbol{x}_{0}, \boldsymbol{x}_{1}, \ldots, \boldsymbol{x}_{k}, \ldots, \boldsymbol{x}_{N} ; \boldsymbol{u}_{0}, \boldsymbol{u}_{1}, \ldots, \boldsymbol{u}_{k}, \ldots, \boldsymbol{u}_{N}\right\}$ are regarded as design variables which are rearranged as vector $\boldsymbol{z}$. Then, terms in Eq.(2) need to be transformed. For differential equations in Eq.(2), in each time interval $\left[t_{k-1}, t_{k}\right], k=1,2, \ldots, N$ they are discretized by the Hermite-Simpson method, which can be expressed as nonlinear equality constraints $\boldsymbol{x}_{k}=\tilde{f}\left(\boldsymbol{x}_{k-1}, \boldsymbol{u}_{k-1}, t\right)$. Thus, differential equations in the time domain $\left[t_{0}, t_{\mathrm{f}}\right]$ are transformed into a series of nonlinear equality constraints. Also considering boundary conditions at time $t_{0}$ and $t_{\mathrm{f}}$, both differential equations and boundary conditions in Eq.(2) are transformed into a series of equality constraints $\overline{\boldsymbol{f}}(\mathbf{z})=\mathbf{0}$. For inequality constraints in Eq.(2), assume they are fulfilled on all grids, all the inequality constraints can be transformed into inequality constraints $\overline{\boldsymbol{g}}(\mathbf{z}) \leq \mathbf{0}$ with respect to $\boldsymbol{z}$. For cost functional in Eq.(2), the HermiteSimpson method is also applied to calculate the integral term, thus it is transformed into a function $y=\boldsymbol{c}(\mathbf{z})$ with respect to $\mathbf{z}$.

Combined all mentioned above, the optimal control problem is transformed into a standard nonlinear programming (NLP):

$$
\begin{gathered}
\min y=\boldsymbol{c}(\mathbf{z}) \\
\text { s.t. } \overline{\boldsymbol{f}}(\mathbf{z})=\mathbf{0} \\
\overline{\boldsymbol{g}}(\mathbf{z}) \leq \mathbf{0}
\end{gathered}
$$

Next, a high effective solver IPOPT[23] is applied to solve the NLP (3) and obtain optimal design variables $\boldsymbol{z}^{*}$. Further, the optimal state variables and control inputs on each grid are obtained.

\section{MODEL CONSTRUCTUON}

\subsection{Luffing mechanism of the hydraulic actuated boom system}

The luffing mechanism is an essential mechanism for a hydraulic actuated boom system. It controls the boom to rotate around the end joint and transfer the linear motion of the cylinder to the rotation motion of the boom. By using luffing mechanism, the hydraulic boom system can be lifted from initial position (zero degree) to almost vertical position. For aerial platform vehicle with folded boom system, the luffing mechanism for the second boom can change the angel between booms to over 150 degree with additional links mechanism.

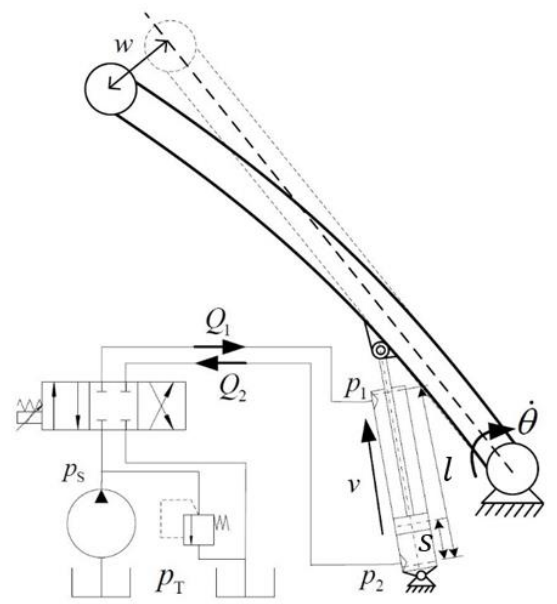

Figure 1: The luffing operation of the hydraulic actuated boom system

As shown in Figure 1, the luffing mechanism can be simplified as a valve-controlled hydraulic cylinder. With stable supply pressure $p_{\mathrm{s}}$, the motion of the cylinder is governed by the in and out volume flow of the both chambers $Q_{1}$ and $Q_{2}$ which can be controlled by the direction valve. The angel velocity $\dot{\theta}$ and angel displacement $\theta$ of 
the boom can be transferred from the piston's velocity of $v$ and displacement $s$ by a simple mechanism kinematic. If we consider the flexibility of the boom structure, an obvious deflection $w$ will occur during the luffing operation if the input from the hydraulic cylinder is not smooth enough.

\subsection{PH model of hydraulic system}

Firstly, we start with the modelling of hydraulic system in port-Hamiltonian formulation. For our case, we consider the luffing operation and the luffing mechanism of the boom system. The main functional component of this hydraulic system is the hydraulic cylinder controlled by directional valve.

The continuity equations of the hydraulic cambers can be described as

$\frac{\mathrm{d}}{\mathrm{d} t}(A s)=Q_{1}, \frac{\mathrm{d}}{\mathrm{d} t}(\alpha A(l-s))=Q_{2}$.

where $A$ is the area of the piston in the non-rod chamber, $\alpha$ is the area ratio between two chambers, $s$ and $l$ is the current displacement and the maximal displacement of the piston, $Q_{1}$ and $Q_{2}$ are the flow rate in and out through the chambers.

Applying the relation of bulk modulus $\beta$ with the properties of oil

$\beta=\rho \frac{\partial p}{\partial \rho}$

the continuity equations can be rewritten as

$\dot{p}_{1}=\frac{\beta}{V_{01}+A s}\left(-A \frac{P_{\mathrm{m}}}{m}+Q_{1}\right)$

$\dot{p}_{2}=\frac{\beta}{V_{02}+\alpha A(l-s)}\left(\alpha A \frac{P_{\mathrm{m}}}{m}-Q_{2}\right)$

with the momentum of the piston $P_{\mathrm{m}}$ and its mass $m$. Combining the dynamic equation of the piston

$\dot{s}=P_{\mathrm{m}} / m$

$\dot{P}_{\mathrm{m}}=p_{1} A-p_{2} \alpha A-F$

we can construct a state model of hydraulic cylinder with the state variable vector as $\lambda=$ $\left[s, P_{\mathrm{m}}, p_{1}, p_{2}\right]^{\mathrm{T}}$.

The energy of the oil inside the both chambers of the cylinder can be described based on the assumption of isentropic fluid as following

$U_{\mathrm{Hydr}}=\sum_{i=1,2} V_{i}\left(\beta e^{\left(p_{i} / \beta\right)}-\beta-p_{i}\right)$

$V_{1}=V_{01}+A s, V_{2}=V_{02}+\alpha A(l-s)$

the subscript number indicate the different chambers. If the kinetic energy of the fluid can be neglected compared to the kinetic energy of the piston, the total energy of the cylinder can be formed as

$E_{\mathrm{c}}=U_{\mathrm{Hydr}}+P_{\mathrm{m}}^{2} / 2 m$.

Using the total energy $E_{\mathrm{c}}$ as the Hamiltonian function of the cylinder $H_{\mathrm{c}}$, the port-Hamiltonian model can be derived with the state vector $\lambda=$ $\left[s, P, p_{1}, p_{2}\right]^{\mathrm{T}}$ and the input vector $\boldsymbol{u}=$ $\left[F, Q_{1}, Q_{2}\right]^{\mathrm{T}}$ as

$\boldsymbol{\lambda}=\boldsymbol{J}(\lambda) \partial_{\lambda} H_{\mathrm{c}}+\boldsymbol{g}(\lambda) \boldsymbol{u}$

$\boldsymbol{y}=\boldsymbol{g}(\lambda)^{\mathrm{T}} \partial_{\lambda} H_{\mathrm{c}}$

The matrices $\boldsymbol{J}(\lambda)$ and $\boldsymbol{g}(\lambda)$ are

$J(\lambda)=\left[\begin{array}{cccc}0 & 1 & 0 & 0 \\ -1 & 0 & \beta A / V_{1} & -\beta \alpha A / V_{2} \\ 0 & -\beta A / V_{1} & 0 & 0 \\ 0 & \beta \alpha A / V_{2} & 0 & 0\end{array}\right]$

$\boldsymbol{g}(\lambda)=\left[\begin{array}{ccc}0 & 0 & 0 \\ -1 & 0 & 0 \\ 0 & \beta / V_{1} & 0 \\ 0 & 0 & -\beta / V_{2}\end{array}\right]$

and the co-energy part is formulated as

$\frac{\partial H_{\mathrm{c}}}{\partial \lambda}=\left[\begin{array}{c}A \Gamma_{1}-\alpha A \Gamma_{2} \\ P_{\mathrm{m}} / m \\ V_{1}\left(\exp \left(p_{1} / \beta\right)-1\right) \\ V_{2}\left(\exp \left(p_{2} / \beta\right)-1\right)\end{array}\right]$,

with $\Gamma_{i}=\beta \exp \left(p_{i} / \beta\right)-\beta-p_{i}, i=1,2$.

The volume flow $Q_{1}$ and $Q_{2}$ can be controlled by the dominate equations of valves as following

$Q_{1}=\left\{\begin{array}{l}k_{v} \sqrt{\left|p_{\mathrm{s}}-p_{1}\right|}\left|x_{v}\right| \operatorname{sgn}\left(p_{\mathrm{s}}-p_{1}\right), x_{v} \geq 0 \\ k_{v} \sqrt{\left|p_{1}-p_{\mathrm{T}}\right|}\left|x_{v}\right| \operatorname{sgn}\left(p_{1}-p_{\mathrm{T}}\right), x_{v}<0\end{array}\right.$

$Q_{2}=\left\{\begin{array}{l}k_{v} \sqrt{\left|p_{2}-p_{\mathrm{T}}\right|}\left|x_{v}\right| \operatorname{sgn}\left(p_{2}-p_{\mathrm{T}}\right), x_{v} \geq 0 \\ k_{v} \sqrt{\left|p_{\mathrm{s}}-p_{2}\right|}\left|x_{v}\right| \operatorname{sgn}\left(p_{\mathrm{s}}-p_{2}\right), x_{v}<0\end{array}\right.$

\subsection{Discretized PH model of rotating Timoshenko beam}

\section{PH formulation}

We simply consider the boom structure as an ideal rotating Timoshenko beam in a plana, when the axial loads can be neglected (due to the relatively small axial deformation). And we started with a rotating homogenous Timoshenko beam formulation to obtain the port-Hamiltonian representation to describe the dynamic behaviour of the boom structure. 
In Figure 2, there is an illustration of a rotating Timoshenko beam with the translational deflection of the beam from the equilibrium position $w(z, t)$ and the rotation of the beam's cross section $\psi(z, t)$.

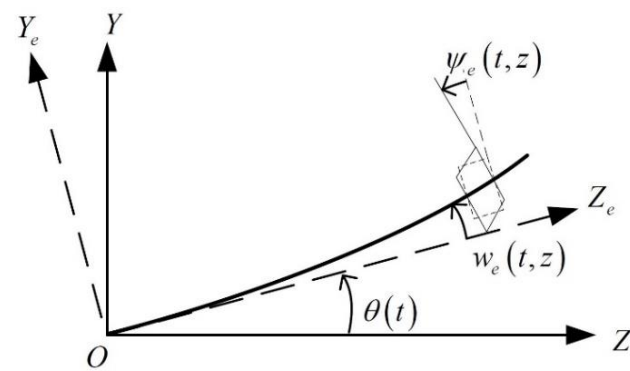

Figure 2: A rotating Timoshenko beam

The boundary condition of such rotating beam can be considered as a free tip with a fixed end, which rotates around an axis resulting an angel displacement $\theta(t)$. Then new definition of the system variables can be given as

$w(z, t)=w_{e}(z, t)+z \cdot \theta(t)$

$\psi(z, t)=\psi_{e}(z, t)+\theta(t)$

which makes the formulation of the rotating Timoshenko beam still fits the original PDEs

$\rho \frac{\partial^{2} w}{\partial t^{2}}-K \frac{\partial^{2} w}{\partial z^{2}}+K \frac{\partial \psi}{\partial z}=0$

$I_{\rho} \frac{\partial^{2} \psi}{\partial t^{2}}-E I \frac{\partial^{2} \psi}{\partial z^{2}}+K\left(\psi-\frac{\partial w}{\partial z}\right)=0$

In equation (12), $\rho$ is the mass per length, $I_{\rho}$ is the mass moment of inertia of the cross section, $E$ is the Young's modulus and $I$ is the moment of the inertia of the cross section. For $K$, there is $K=k G A$ and $G$ is the modulus of elasticity in shear, $A$ is the area of cross section and $k$ is a constant depending on the shape of the cross section.

Combining the kinetic energy and elastic potential energy, we can give the Hamiltonian function of a Timoshenko beam as following:

$H_{\mathrm{B}}(t)=\frac{1}{2} \int_{0}^{L}\left(\rho \dot{w}^{2}+I_{\rho} \dot{\psi}^{2}+K\left(\psi-\partial_{z} w\right)^{2}+\right.$

$\left.E I\left(\partial_{z} \psi\right)^{2}\right) \mathrm{d} z$

The kinetic energy is the function of the translational and rotational momenta which are given as

$$
\begin{aligned}
& p_{\mathrm{t}}(z, t)=\rho \dot{w}(z, t) \\
& p_{\mathrm{r}}(z, t)=I_{\rho} \dot{\psi}(z, t)
\end{aligned}
$$

and the co-energy variables are translational velocity and rotational velocity. The elastic potential energy is the function of the shear and bending deformation which are defined as

$\varepsilon_{\mathrm{t}}(z, t)=\partial_{z} w(z, t)-\psi(z, t)$

$\varepsilon_{\mathrm{r}}(z, t)=\partial_{z} \psi(z, t)$

and the co-energy variables are shear force and bending momentum.

According to the definition of new state variables, the original PDEs of Timoshenko beam can be rewritten as

$\dot{\boldsymbol{x}}=\left[\begin{array}{c}\dot{p}_{\mathrm{t}} \\ \dot{p}_{\mathrm{r}} \\ \dot{\varepsilon}_{\mathrm{t}} \\ \dot{\varepsilon}_{\mathrm{r}}\end{array}\right]=\left[\begin{array}{cccc}0 & 0 & \partial_{z} & 0 \\ 0 & 0 & 1 & \partial_{z} \\ \partial_{z} & -1 & 0 & 0 \\ 0 & \partial_{z} & 0 & 0\end{array}\right]\left[\begin{array}{c}\delta_{p_{\mathrm{t}}} H \\ \delta_{p_{\mathrm{r}}} H \\ \delta_{\varepsilon_{\mathrm{t}}} H \\ \delta_{\varepsilon_{\mathrm{r}}} H\end{array}\right]$

We denote $\boldsymbol{e} \in \mathcal{E}, \boldsymbol{f} \in \mathcal{F}$ as the effort and flow variables separately. They are related with the time derivative of state variables and the associated co-energy variables as

$\boldsymbol{f}=\left[\begin{array}{l}f^{p_{\mathrm{t}}} \\ f^{p_{\mathrm{r}}} \\ f^{\varepsilon_{\mathrm{t}}} \\ f^{\varepsilon_{\mathrm{r}}}\end{array}\right]=-\left[\begin{array}{c}\dot{p}_{\mathrm{t}} \\ \dot{p}_{\mathrm{r}} \\ \dot{\varepsilon}_{\mathrm{t}} \\ \dot{\varepsilon}_{\mathrm{r}}\end{array}\right], \boldsymbol{e}=\left[\begin{array}{c}e^{p_{\mathrm{t}}} \\ e^{p_{\mathrm{r}}} \\ e^{\varepsilon_{\mathrm{t}}} \\ e^{\varepsilon_{\mathrm{r}}}\end{array}\right]=\left[\begin{array}{c}\delta_{p_{\mathrm{t}} H} \\ \delta_{p_{\mathrm{r}}} H \\ \delta_{\varepsilon_{\mathrm{t}}} H \\ \delta_{\varepsilon_{\mathrm{r}}} H\end{array}\right]$

The change of the total energy of the beam (quadratic energy only, ignore the gravity potential energy) is expressed as

$\dot{H}_{\mathrm{B}}=\int_{0}^{L}\left(\partial_{x} H\right)^{\mathrm{T}} \dot{\boldsymbol{x}} \mathrm{d} z=-\int_{0}^{L} \boldsymbol{e}^{\mathrm{T}} \boldsymbol{f} \mathrm{d} z$.

By applying integration by parts, the change of the total energy can also be illustrated as the variable pairs of power through the boundaries as

$\dot{H}_{\mathrm{B}}=e^{p_{\mathrm{t}}} e^{\varepsilon_{\mathrm{t}}}+\left.e^{p_{\mathrm{r}}} e^{\varepsilon_{\mathrm{r}}}\right|_{0} ^{L}$

and we define the boundary flow and effort as

$\left[\begin{array}{l}f_{\partial}^{\mathrm{t}} \\ f_{\partial}^{\mathrm{r}} \\ e_{\partial}^{\mathrm{t}} \\ e_{\partial}^{\mathrm{r}}\end{array}\right]=\left[\begin{array}{l}\left.e^{p_{\mathrm{t}}}\right|_{\partial_{z}} \\ \left.e^{p_{\mathrm{r}}}\right|_{\partial_{z}} \\ \left.e^{\varepsilon_{\mathrm{t}}}\right|_{\partial_{z}} \\ \left.e^{\varepsilon_{\mathrm{t}}}\right|_{\partial_{z}}\end{array}\right]$

where $\partial_{Z}$ denotes the boundary of the domain $Z=[0, L]$. And the power continuity and conservation equation are fulfilled as

$\int_{Z} \boldsymbol{e}^{\mathrm{T}} \cdot \boldsymbol{f} \mathrm{d} z+\left.\left(f_{\partial}^{\mathrm{t}} \cdot e_{\partial}^{\mathrm{t}}+f_{\partial}^{\mathrm{r}} \cdot e_{\partial}^{\mathrm{r}}\right)\right|_{\partial Z}=0$

Finally, the equation can be simplified as

$-\boldsymbol{f}=\boldsymbol{J}(z) \partial_{x} H_{\mathrm{B}}=\boldsymbol{J}(z) \boldsymbol{e}$ 
where $\boldsymbol{J}(z)$ is a skew-symmetric differential operator and further discretization method should be applied to solve the PDEs.

\section{Structure-preserving discretization}

The next step to solve the PDEs of Timoshenko beam is to transfer into a discretized model. And the skew-symmetric differential operator must be retained in the new model, which means that the certain geometric or structure property should be preserved after the discretization. In [10], Moulla applied a structure-preserving discretization method based on pseudo-spectral method to approximate the infinite-dimensional Timoshenko beam model.

The discretization of the differential operator $J(z)$ has proven as a good approximation of the properties of the system in [13]. We list some essential steps of the discretization in this section.

In the formulation (22), the differential operator $J(z)$ and the effort vector can be separated as two parts by the subject relation to differentiation. The new effort vectors are denoted as $\boldsymbol{e}$ and $\boldsymbol{e}_{*}$ and the new formulation is expressed as

$-\boldsymbol{f}=\left[\begin{array}{cccc} & & 1 & 0 \\ 1 & 0 & & 1 \\ 0 & 1 & \end{array}\right] \partial_{z} \boldsymbol{e}+\left[\begin{array}{cccc} & & 0 & 0 \\ & & 1 & 0 \\ 0 & -1 & & \end{array}\right] \boldsymbol{e}_{*}$.

According to the pseudo-spectral method proposed for canonical system of two conservation laws, we define different approximation bases for the flows $\boldsymbol{f}^{v} \in$ $\left\{\boldsymbol{f}^{p_{\mathrm{t}}}, \boldsymbol{f}^{p_{\mathrm{r}}}, \boldsymbol{f}^{\varepsilon_{\mathrm{t}}}, \boldsymbol{f}^{\varepsilon_{\mathrm{r}}}\right\}$ and the efforts $\boldsymbol{e}^{v} \in$ $\left\{\boldsymbol{e}^{p_{\mathrm{t}}}, \boldsymbol{e}^{p_{\mathrm{r}}}, \boldsymbol{e}^{\varepsilon_{\mathrm{t}}}, \boldsymbol{e}^{\varepsilon_{\mathrm{r}}}\right\}$.

$\boldsymbol{f}^{v}(t, z) \approx \sum_{k=0}^{N-1} f_{k}^{v} \varphi_{k}(z)$,

$\boldsymbol{e}_{*}^{v}(t, z) \approx \sum_{K=0}^{N-1} e_{*}^{v} \varphi_{k}(z)$,

$\boldsymbol{e}^{v}(t, z) \approx \sum_{i=0}^{N} e^{v} \phi_{i}(z)$

$\varphi_{k}(z)$ and $\phi_{i}(z)$ are the basis functions for flows and efforts. We chose the interpolation Lagrange polynomials of degree $N$ and $N-1$ as suitable basis functions:

$\varphi_{k}(z)=\prod_{j=0, j \neq k}^{N-1} \frac{z-z_{j}}{z_{k}-z_{j}}, \phi_{i}(z) \prod_{j=0, j \neq i}^{N} \frac{z-\xi_{j}}{\xi_{i}-\xi_{j}}$

with $\quad z_{k} \in(0, L), k=0, \ldots, N-1 \quad$ and $\quad \xi_{i} \in$ $(0, L), i=0, \ldots, N$ are the collocation points for $\varphi_{k}(z)$ and $\phi_{i}(z)$ respectively.

We directly give the discretized formulation of a rotating Timoshenko beam as an input-output representation: $\overline{\boldsymbol{f}}=\left[\begin{array}{cc}0 & \boldsymbol{J}_{12} \\ \boldsymbol{J}_{21} & 0\end{array}\right] \cdot \overline{\boldsymbol{e}}$

with

$\overline{\boldsymbol{f}}=\left[\boldsymbol{f}^{p_{\mathrm{t}}},-e_{\partial 0}^{\mathrm{t}}, \boldsymbol{f}^{p_{\mathrm{r}}},-e_{\partial 0}^{\mathrm{r}}, \boldsymbol{f}^{\varepsilon_{\mathrm{t}}}, f_{\partial L}^{\mathrm{t}}, \boldsymbol{f}^{\varepsilon_{\mathrm{r}}}, f_{\partial L}^{\mathrm{r}}\right]^{\mathrm{T}}$

$\overline{\boldsymbol{e}}=\left[\tilde{\boldsymbol{e}}^{p_{\mathrm{t}}}, f_{\partial 0}^{\mathrm{t}}, \tilde{\boldsymbol{e}}^{p_{\mathrm{r}}}, f_{\partial 0}^{\mathrm{r}}, \tilde{\boldsymbol{e}}^{\varepsilon_{\mathrm{t}}}, e_{\partial L}^{\mathrm{t}}, \tilde{\boldsymbol{e}}^{\varepsilon_{\mathrm{r}}}, e_{\partial L}^{\mathrm{r}}\right]^{\mathrm{T}}$

The details inside $\boldsymbol{J}_{12}$ and $\boldsymbol{J}_{21}$ are given in the previous work [24], and it can be proven that $\boldsymbol{J}_{12}=-\boldsymbol{J}_{21}^{\mathrm{T}}$, i.e. the interconnection matrix $\boldsymbol{J} \in$ $\mathbb{R}^{(4 N+4) \times(4 N+4)}$ is skew-symetric.

$$
\begin{aligned}
& \boldsymbol{\phi}_{0}=\left[\phi_{0}(0), \ldots, \phi_{N}(0)\right]^{\mathrm{T}} \\
& \boldsymbol{\phi}_{L}=\left[\phi_{0}(L), \ldots, \phi_{N}(L)\right]^{\mathrm{T}} \\
& \boldsymbol{D} \in \mathbb{R}^{N \times(N+1)}, D_{k+1, i+1}=\partial_{z} \phi_{i}\left(z_{k}\right) \\
& \boldsymbol{M} \in \mathbb{R}^{(N+1) \times N}, M_{i+1, k+1}=\int_{0}^{L} \phi_{i}(z) \varphi_{k}(z) \mathrm{d} z \\
& \boldsymbol{S} \in \mathbb{R}^{N \times N}, S_{i+1 j+1}=\int_{0}^{L} \varphi_{i}(z) \varphi_{j}(z) \mathrm{d} z
\end{aligned}
$$

Finally, we obtain an explicit linear portHamiltonian formulation with input and output as

$$
\begin{aligned}
& \dot{\boldsymbol{X}}=\boldsymbol{J}_{4 N \times 4 N} \boldsymbol{Q}_{4 N \times 4 N} \boldsymbol{X}+\boldsymbol{G}_{4 N \times 4} \boldsymbol{U} \\
& \boldsymbol{Y}=\boldsymbol{G}_{4 N \times 4}^{\mathrm{T}} \boldsymbol{Q}_{4 N \times 4 N} \boldsymbol{X}+\boldsymbol{D}_{4 \times 4} \boldsymbol{U}
\end{aligned}
$$

The discretized flow vectors are remained as state variables $\boldsymbol{X} \in \mathbb{R}^{4 N}$.The properties of the cross section is reflected by the matrix $\boldsymbol{Q}_{4 N \times 4 N}=$ blockdiag $\left\{\boldsymbol{S} / \rho, \boldsymbol{S} / I_{\rho}, K_{s} \boldsymbol{S}, K_{b} \boldsymbol{S}\right\}$. The boundary flows efforts are relocated in the input and output vectors as

$$
\boldsymbol{U}(\boldsymbol{t})=\left[\begin{array}{c}
f_{\partial 0}^{\mathrm{t}} \\
f_{\partial 0}^{\mathrm{r}} \\
e_{\partial L}^{\mathrm{t}} \\
e_{\partial L}^{\mathrm{r}}
\end{array}\right]=\left[\begin{array}{c}
v(0) \\
\omega(0) \\
Q(L) \\
M(L)
\end{array}\right], \boldsymbol{Y}(t)=\left[\begin{array}{c}
-e_{\partial 0}^{\mathrm{t}} \\
-e_{\partial 0}^{\mathrm{r}} \\
f_{\partial L}^{\mathrm{t}} \\
f_{\partial L}^{\mathrm{r}}
\end{array}\right]=\left[\begin{array}{c}
-Q(0) \\
-M(0) \\
v(L) \\
\omega(L)
\end{array}\right]
$$

\subsection{Model assembly}

Between the hydraulic cylinder and the boom structure, there is the luffing mechanism to connect both sides and to transfer motion and load. We specify the relation between the displacement of cylinder $s$ and the angel displacement of the boom $\theta$ as $\theta(s)$, the relation of load and velocity can be expressed as

$$
\begin{aligned}
& F=\partial_{s} \theta \cdot M(0) \\
& \omega(0)=\partial_{s} \theta \cdot \dot{s}
\end{aligned}
$$

Now we can connect the two system with the relation between the input and output. We found the matrix $\boldsymbol{G}_{4 N \times 4}$ in the formulation of boom model can be written as 
$\boldsymbol{G}_{4 N \times 4}=\left[\begin{array}{cccc}0 & 0 & \boldsymbol{G}_{0} & 0 \\ 0 & 0 & 0 & \boldsymbol{G}_{0} \\ \boldsymbol{G}_{0} & 0 & 0 & 0 \\ 0 & \boldsymbol{G}_{0} & 0 & 0\end{array}\right]$

with $\boldsymbol{G}_{0}$ as $1 \times N$ vector.

We can list the equations of both systems together with the details of interconnection terms as

$$
\left[\begin{array}{c}
\dot{\lambda} \\
\boldsymbol{f}^{p_{\mathrm{t}}} \\
\boldsymbol{f}^{p_{\mathrm{r}}} \\
\boldsymbol{f}^{\varepsilon_{\mathrm{t}}} \\
\boldsymbol{f}^{\varepsilon_{\mathrm{r}}}
\end{array}\right]=\left[\begin{array}{cc}
4 \times 4 & \\
\overbrace{\boldsymbol{J}(\lambda)} & 0 \\
0 & \underbrace{J(X)}_{4 N \times 4 N}
\end{array}\right]\left[\begin{array}{c}
\partial_{\lambda} H_{\mathrm{c}} \\
\tilde{\boldsymbol{e}}^{p_{\mathrm{t}}} \\
\tilde{\boldsymbol{e}}^{p_{\mathrm{r}}} \\
\tilde{\boldsymbol{e}}^{\varepsilon_{\mathrm{t}}} \\
\tilde{\boldsymbol{e}}^{\varepsilon_{\mathrm{r}}}
\end{array}\right]+\left[\begin{array}{c}
0 \\
\partial_{s} \theta \boldsymbol{G}_{0}^{\mathrm{T}} \tilde{\boldsymbol{e}}^{\varepsilon_{\mathrm{r}}} \\
\beta Q_{1} / V_{1} \\
-\beta Q_{2} / V_{2} \\
Q(L) \boldsymbol{G}_{0} \\
M(L) \boldsymbol{G}_{0} \\
v(0) \boldsymbol{G}_{0} \\
-\partial_{S} \theta \boldsymbol{G}_{0} \frac{P_{\mathrm{m}}}{m}
\end{array}\right]
$$

Then a complete model of the hydraulic actuated boom system can be formed with the new definition of the state yariables as $\dot{\bar{X}}=$

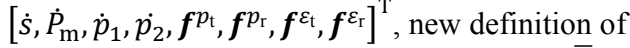
the input vector as $\overline{\boldsymbol{U}}=$ $\left[Q_{1}, Q_{2}, Q(L), M(L), v(0)\right]^{\mathrm{T}}$ and the Hamiltonian function of the complete system as $H=H_{\mathrm{c}}+$ $H_{\mathrm{B}}$. The equations for the complete system can be expressed as

$$
\dot{\overline{\boldsymbol{X}}}=\overline{\boldsymbol{J}}(\overline{\boldsymbol{X}}) \partial_{\overline{\boldsymbol{X}}} H+\overline{\boldsymbol{G}} \overline{\boldsymbol{U}}
$$

with the detail of $\overline{\boldsymbol{J}}(\overline{\boldsymbol{X}})$ as following:

$$
\left[\begin{array}{cccccccc}
0 & 1 & 0 & 0 & 0 & 0 & 0 & 0 \\
-1 & 0 & \mu_{1} & -\mu_{2} & 0 & 0 & 0 & \partial_{S} \theta \boldsymbol{G}_{0}^{\mathrm{T}} \\
0 & -\mu_{1} & 0 & 0 & 0 & 0 & 0 & 0 \\
0 & \mu_{2} & 0 & 0 & 0 & 0 & 0 & 0 \\
0 & 0 & 0 & 0 & 0 & 0 & \boldsymbol{J}_{13} & 0 \\
0 & 0 & 0 & 0 & 0 & 0 & \boldsymbol{J}_{23} & \boldsymbol{J}_{24} \\
0 & 0 & 0 & 0 & \boldsymbol{J}_{31} & \boldsymbol{J}_{32} & 0 & 0 \\
0 & -\partial_{S} \theta \boldsymbol{G}_{0} & 0 & 0 & 0 & \boldsymbol{J}_{42} & 0 & 0
\end{array}\right] .
$$

\section{SOLUTION OF THE OPTIMAL CONTROL PROBLEM}

In order to solve the optimal control problem related to the hydraulic cylinder, we firstly applied the nondimensionalization method proposed by Sakai[19].

With the definition of the new nondimensionalized state variables $s=s^{*} l, P=$ $P^{*} \sqrt{m l \beta A}, t=t^{*} T=t^{*} \sqrt{m l \beta A}, p_{1}=\beta p_{1}^{*}$, $p_{2}=\beta p_{2}^{*}$, the original equations of the hydraulic cylinder (6) and can be reformed as

$$
\begin{aligned}
& \frac{d s^{*}}{d t^{*}}=P^{*} \\
& \frac{d P^{*}}{d t^{*}}=p_{1}^{*}-p_{2}^{*}-\frac{F}{\beta A} \\
& \frac{d p_{1}^{*}}{d t^{*}}=\frac{-P^{*} l+\frac{Q_{1}}{A} \sqrt{m l \beta A}}{V_{01} / A+l s^{*}} \\
& \frac{d p_{2}^{*}}{d t^{*}}=\frac{P^{*} l-\frac{Q_{2}}{A} \sqrt{m l \beta A}}{V_{02} / A+\alpha l\left(l-s^{*}\right)}
\end{aligned}
$$

In the optimal control problem, the state variables are nondimensionalized variables $\lambda^{*}=$ $\left[s^{*}, P^{*}, p_{1}^{*}, p_{2}^{*}\right]$. The differential equations are Eq.(29).

The cost functional is set as:

$J=\int_{0}^{t_{f}}\left(Q_{1}^{2}+Q_{2}^{2}\right) \times 10^{5}+\left(p_{1}^{* 2}+p_{2}^{* 2}\right)$

$\times 10^{6} \mathrm{~d} t+P^{* 2} \times 10^{2}$

where simulation time is set as $t_{\mathrm{f}}=0.76 \mathrm{~s}$.

At initial instant, both the displacement and the velocity of cylinder are set as 0 . Pressure $p_{1}$ and pressure $p_{2}$ are set as $5 \times 10^{5} \mathrm{~Pa}$ and 0 , respectively. At terminal instant, the position of cylinder is $l_{\max }=0.3935 \mathrm{~m}$. The velocity of cylinder is 0 . Pressure $p_{1}$ and pressure $p_{2}$ are set as $5 \times 10^{5} \mathrm{~Pa}$ and 0 , respectively.

Thus, the initial conditions and terminal conditions in optimal control problem are set as:

$$
\begin{aligned}
& \lambda_{0}^{*}=\left[\begin{array}{lll}
0 & 0 & \frac{5 \times 10^{5}}{\beta} 0
\end{array}\right]^{\mathrm{T}}, \\
& \lambda_{\mathrm{f}}^{*}=\left[l_{\max } / l 05 \times 10^{5} / \beta 0\right]^{\mathrm{T}}
\end{aligned}
$$

During the movement, pressure $p_{1}$ and pressure $p_{2}$ should be great than zeros. The rate of momentum also should be limited. One has the following inequality constraints:

$\lambda_{3}^{*} \geq 0, \lambda_{4}^{*} \geq 0,-\lambda_{\max }^{*} \leq \dot{\lambda}_{2}^{*} \leq \lambda_{\max }^{*}$

In this example, the threshold is set as $\lambda_{\max }^{*}=$ $3 \times 10^{-4}$.

Combining Eq.(29)-Eq.(32), an optimal control problem is defined properly. ICLOCS 2.5 is applied to solve the problem. The converged solution is obtained after 500 iterations which take $20.43 \mathrm{~s}$. The cost functional is $1.1897 \times 10^{4}$.

The cost function means the minimal input and the potential energy. All the parameters we use are listed in Table 1.

Table 1: Parameters of the cylinder

\begin{tabular}{ll}
\hline Cylinder parameter & Value \\
\hline Cap end area $A$ & $1 \times 10^{-4} \mathrm{~m}^{2}$ \\
Area ratio $\alpha$ & 0.75 \\
Bulk modulus $\beta$ & $1.29 \times 10^{9} \mathrm{~Pa}$
\end{tabular}




\begin{tabular}{ll} 
Initial volume in chamber $1 V_{01}$ & $1 \times 10^{-7} \mathrm{~m}^{3}$ \\
Initial volume in chamber $2 V_{02}$ & $1 \times 10^{-7} \mathrm{~m}^{3}$ \\
Stroke of the cylinder $l$ & $0.5 \mathrm{~m}$ \\
Mass of the piston $m$ & $10 \mathrm{~kg}$ \\
Force on the piston $F$ & $50 \mathrm{~N}$ \\
\hline
\end{tabular}

We made a test simulation for a single hydraulic cylinder to be driven to a maximal displacement of $0.3 \mathrm{~m}$ in a short time period of $0.765 \mathrm{~s}$ (it comes from the nondimensionalized time variable). The results of state variables and inputs are shown in Figure 3 to Figure 6.

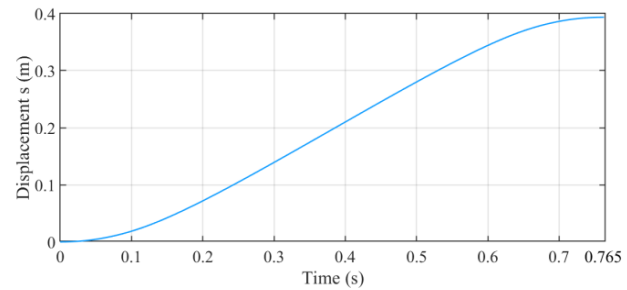

Figure 3: Optimal result of displacement $s$

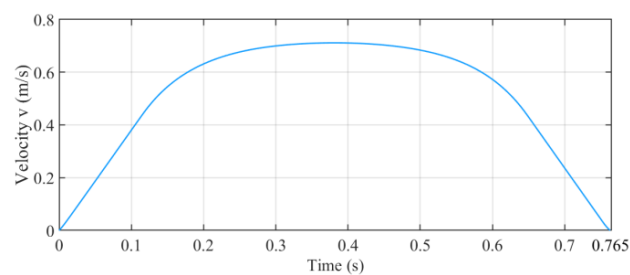

Figure 4: Optimal result of piston velocity $v$

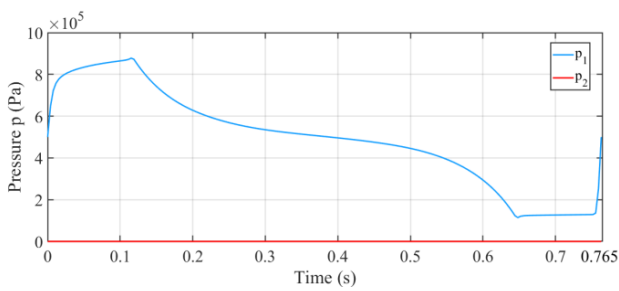

Figure 5: Optimal results of pressures $p_{1}$ and $p_{2}$

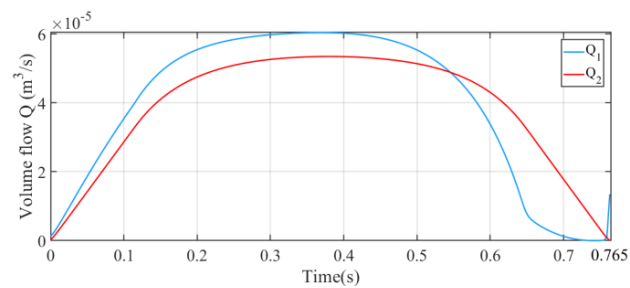

Figure 6: Optimal results of volume flows $Q_{1}$ and $Q_{2}$

The pressure $p_{2}$ in chamber 2 is calculated as 0 for the whole time period. It is reasonable because of the absent of the equation of control valve as Eq.(10), it means there is no throttling effect between chamber 2 and the tank. It reveals that only using volume flows as controlled inputs is not good enough for the solution of the optimal control problem of hydraulic cylinder. This also points out an improvement for us.

Next, we apply the piston velocity $v$ combining with the Eq.(28) to calculate the corresponding input angel velocity for the inputoutput system of boom structure as (27). The parameters of the boom structure we designed are listed in Table 2 which are modified from our previous model in [24].

Table 2: Parameters of the boom structure

\begin{tabular}{llll}
\hline Parameter & Beam 1 & Beam 2 & Beam 3 \\
\hline Length (m) & & 0.3 & \\
Width (m) & 0.2 & 0.15 & 0.1 \\
Depth (m) & 0.005 & 0.004 & 0.003 \\
Density (kg/m $\left.{ }^{3}\right)$ & & 7850 & \\
Yong's modulus (GPa) & 210 & \\
Poisson's ratio & 0.33 & \\
Shear factor & $5 / 6$ & \\
\hline
\end{tabular}

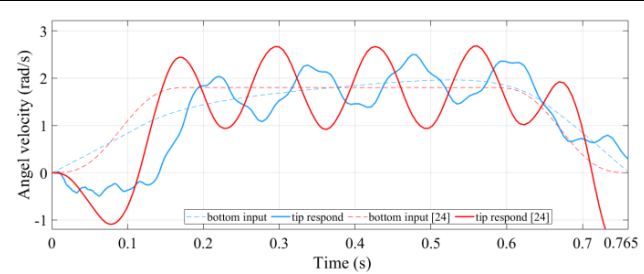

Figure 7: The angel velocity inputs, and the angel velocity outputs of two cases

The boom structure is modelled as a step beam with three different sections. The input is the angel velocity at the bottom of the boom and the output is the angel velocity at the tip of the boom. In Figure 7, the dot lines are the input curves of optimized angel velocity (blue) and the one (red) we applied in [24] generated by quadratic function. The solid lines are the corresponding outputs. The results present the improvement of less vibration amplitude between optimized input and the previous input. But the reduce of the vibration is not significant due to the lack of boom model in the optimization process. 


\section{CONCLUSION AND OUTLOOK}

In this paper, we present a complete model of hydraulic actuated boom system in the formulation of port-Hamiltonian system. It is a foundation step to apply some dynamic control method to control the vibration of the flexible boom structure during the luffing operation. We also develop an offline trajectory generation method based on optimal control problem to generate the optimal motion of the hydraulic cylinder based on the energy related cost function.

There are still some parts of our work needed to be improved in the future, such as to include the dominate equations of control valve and the linear boom structure model in the formulation of the optimal control problem. An online model predictive control strategy will be investigated to solve the real-time vibration control problem in the next step.

\section{NOMENCLATURE}

PH Port-Hamiltonian

NLP Nonlinear programming

\section{REFERENCES}

[1]. Sun, G., \& Kleeberger, M. (2003). Dynamic responses of hydraulic mobile crane with consideration of the drive system. Mechanism and Machine Theory, 38(12), 1489-1508.

[2]. Sun, G., Kleeberger, M., \& Liu, J. (2005). Complete dynamic calculation of lattice mobile crane during hoisting motion. Mechanism and machine theory, 40(4), 447-466.

[3]. Sun, G., \& Liu, J. (2006). Dynamic responses of hydraulic crane during luffing motion. Mechanism and Machine Theory, 41(11), 12731288.

[4]. Zuyev, A., \& Sawodny, O. (2005). Stabilization of a flexible manipulator model with passive joints. IFAC Proceedings Volumes, 38(1), 784789.

[5]. Sawodny, O., Aschemann, H., \& Bulach, A. (2002). Mechatronical designed control of firerescue turntable-ladders as flexible link robots. IFAC Proceedings Volumes, 35(1), 509-514.

[6]. Pertsch, A., Zimmert, N., \& Sawodny, O. (2009, December). Modeling a fire-rescue turntable ladder as piecewise Euler-Bernoulli beam with a tip mass. In Proceedings of the $48 \mathrm{~h}$ IEEE Conference on Decision and Control (CDC) held jointly with 2009 28th Chinese Control Conference (pp. 7321-7326). IEEE.

[7]. Pertsch, A., \& Sawodny, O. (2016). Modelling and control of coupled bending and torsional vibrations of an articulated aerial ladder. Mechatronics, 33, 34-48.

[8]. Duindam, V., Macchelli, A., Stramigioli, S., \& Bruyninckx, H. (2009). Modeling and control of complex physical systems: The portHamiltonian approach. Springer Science \& Business Media.

[9]. Macchelli, A. \& Melchiorri, C. (2004). Modeling and control of the Timoshenko beam. the distributed port Hamiltonian approach. SIAM Journal on Control and Optimization, 43(2):743-767.

[10].Macchelli, A., Melchiorri, C., \& Stramigioli, S. (2009). Port-based modeling and simulation of mechanical systems with rigid and flexible links. IEEE transactions on robotics, 25(5):10161029 .

[11].Moulla, R., Lefevre, L., \& Maschke, B. (2012). Pseudo- spectral methods for the spatial symplectic reduction of open systems of conservation laws. Journal of computational Physics, 231(4):1272-1292.

[12].Vu, N. M. T., Lefevre, L., Nouailletas, R., \& Bre'mond, S. (2013). Geometric discretization for a plasma control model. IFAC Proceedings Volumes, 46(2):755-760.

[13].Wang, M., Bestler, A., \& Kotyczka, P. (2017). Modeling, discretization and motion control of a flexible beam in the port-Hamiltonian framework. IFAC- Papers On Line, 50(1):67996806.

[14].Kugi, A. \& Kemmetmüller, W. (2004). New energy- based nonlinear controller for hydraulic piston actuators. European journal of control, 10(2):163-173.

[15].Grabmair, G., \& Schlacher, K. (2005) Energybased nonlinear control of hydraulically actuated mechanical systems. In Proceedings of the 44th IEEE Conference on Decision and Control (pp. 7520-7525). IEEE.

[16].Sakai, S., \& Stramigioli, S. (2009). Passivity based force control of hydraulic robots. IFAC Proceedings Volumes, 42(16), 20-25.

[17].Sakai, S. (2010). Passivity based control of hydraulic linear arms using natural Casimir functions. In Cutting Edge Robotics 2010. IntechOpen

[18].Sakai, S. (2013). Casimir based fast computation for hydraulic robot optimizations. In 2013 
IEEE/RSJ International Conference on Intelligent Robots and Systems (pp. 2874-2881). IEEE.

[19].Sakai, S. (2017, May). A structure preserving nondimensionalization of hydraulic rotational joints. In 2017 IEEE International Conference on Robotics and Automation (ICRA) (pp. 26-32). IEEE.

[20].Stadlmayr, R., \& Schlacher, K. (2004). Modelling and Control of a Hydraulic Actuated Large Scale Manipulator. In PAMM: Proceedings in Applied Mathematics and Mechanics (Vol. 4, No. 1, pp. 141-142). Berlin: WILEY- VCH Verlag.

[21].Nie, Y., Faqir, O., \& Kerrigan, E. C. (2018, September). ICLOCS2: Try this Optimal Control Problem Solver Before you Try the Rest. In 2018 UKACC 12th International Conference on Control (CONTROL) (pp. 336-336). IEEE.

[22].Nie, Y., Faqir, O., \& Kerrigan, E. C. (2018). ICLOCS2: Solve your optimal control problems with less pain.

[23].Waechter, A., Laird, C., Margot, F., \& Kawajir, Y. (2009). Introduction to IPOPT: A tutorial for downloading, installing, and using IPOPT. Revision.

[24].Gao, L., Mei, W., Kleeberger, M., Peng, H., \& Fottner, J. (2019). Modeling and Discretization of Hydraulic Actuated Telescopic Boom System in Port-Hamiltonian Formulation. In Proceedings of the 9th International Conference on Simulation and Modeling Methodologies, Technologies and Applications (pp. 69-79). SCITEPRESS-Science and Technology Publications, Lda. 